3. W. Sierpinski, General topology, Univ. of Toronto Press, Ontario, 1952, p. 250.

4. - Cardinal and ordinal numbers, PWN, Warsaw, 1958, p. 376.

5. S. M. Ulam, Zur Masstheorie in der allgemeinen Mengenlehre, Fund. Math. 16 (1930), 140.

6. - Problèmes No. 74, Fund. Math. 30 (1938), 365.

7. - A collection of mathematical problems, Interscience, New York, 1960, p. 9.

Indian Statistical Institute, Calcutta, India

\title{
ON SPHERE-BUNDLES. I
}

BY I. M. JAMES ${ }^{1}$

Communicated by P. E. Thomas, November 19, 1968

Let $E$ be an $(n-1)$-sphere bundle over a base space $B$, with the orthogonal group as structural group. By an almost-complex structure on $E$ we mean a reduction of the structural group to the unitary group. By an $A$-structure on $E$ I mean a fibre-preserving map $f: E \rightarrow E$ such that $f x$ is orthogonal to $x$ for all $x \in E$. For example, an almostcomplex structure determines such a map through the action ${ }^{2}$ of the scalar $J$ such that $J^{2}=-1$. Note that $n$ must be even if an $A$-structure exists. When $E$ is trivial this necessary condition is also sufficient.

I describe $E$ as homotopy-symmetric if $1 \cong u: E \rightarrow E$, by a fibrepreserving homotopy, where $u$ denotes the antipodal map given by $u x=-x$. This condition also implies that $n$ is even. An $A$-structure $f$ on $E$ determines a fibre-preserving homotopy $f_{t}(t \in I=[0,1])$, where $f_{t} x=x \cos \pi t+f(x) \sin \pi t$, and so $E$ is homotopy-symmetric. I assert that the converse holds in the stable range ${ }^{3}$ so that we have

TheOREM 1. Let $B$ be a finite complex such that $\operatorname{dim} B \leqq n-4$. Then $E$ admits an $A$-structure if and only if $E$ is homotopy-symmetric.

A proof can be given as follows. Let $p: E \rightarrow B$ denote the fibration. Let $E^{\prime}$ denote the space of pairs $(x, y)$, where $x, y \in E$, such that $p x=p y$ and such that $x$ is orthogonal to $y$. We fibre $E^{\prime}$ over $E$ with projection $p^{\prime}$ given by $p^{\prime}(x, y)=x$. An $A$-structure $f$ on $E$ determines a cross-section $f^{\prime}: E \rightarrow E^{\prime}$, where $f^{\prime} x=(x, f x)$, and conversely a crosssection determines an $A$-structure. Let $E^{\prime \prime}$ denote the space of paths $\lambda$ in $E$ such that $p \lambda$ is stationary in $B$ and such that $\lambda(0)=\lambda(1)$. We

1 Research partly supported by the National Science Foundation.

2 We recall that the centre of the structural group acts on the bundle.

${ }^{3}$ The stable range, in relation to this problem, is not quite as extensive as the stable range of ordinary theory. 
fibre $E^{\prime \prime}$ over $E$ with projection $p^{\prime \prime}$ given by $p^{\prime \prime} \lambda=\lambda(0)$. Then $p^{\prime \prime} h=p^{\prime}$, where $h: E^{\prime} \rightarrow E^{\prime \prime}$ is the map defined by

$$
h(x, y)(t)=x \cos \pi t+y \sin \pi t \quad(t \in I) .
$$

A fibre-homotopy $f_{t}$ of 1 into $u$ determines a cross-section $f^{\prime \prime}: E \rightarrow E^{\prime \prime}$, where $f^{\prime \prime}(x)(t)=f_{t}(x)$, and conversely.

Consider the fibre $S^{n-1}$ of the original fibration $p: E \rightarrow B$ which contains the basepoint $e \in E$. The fibre of $p^{\prime}: E^{\prime} \rightarrow E$ over $e$ can be identified with $S^{n-2}$, the equator orthogonal to $e$. The corresponding fibre of $p^{\prime \prime}: E^{\prime \prime} \rightarrow E$ can be identified with $\Omega\left(S^{n-1}\right)$, the space of paths in $S^{n-1}$ from $e$ to $-e$, so that $h$ maps $x \in S^{n-2}$ into the great semicircle through $x$. It follows that the Freudenthal suspension can be expressed as the composition

$$
\pi_{r}\left(S^{n-2}\right) \underset{h_{*}}{\rightarrow} \pi_{r}\left(\Omega\left(S^{n-1}\right)\right) \underset{\theta}{\rightarrow} \pi_{r+1}\left(S^{n-1}\right),
$$

where $\theta$ denotes the Hurewicz isomorphism. Therefore $h_{*}$ is injective for $r<2 n-5$ and surjective for $r \leqq 2 n-5$. However

$$
\operatorname{dim} E=n-1+\operatorname{dim} B \leqq 2 n-5,
$$

by hypothesis, and so the coefficient homomorphism

$$
H^{i}\left(E, \pi_{j}\left(S^{n-2}\right)\right) \rightarrow H^{i}\left(E, \pi_{j}\left(\Omega\left(S^{n-1}\right)\right)\right), \cdot
$$

induced by $h_{*}$ is injective for $j<i$, surjective for $j \leqq i$. Hence it follows by obstruction theory, as in [2], that $p^{\prime}: E^{\prime} \rightarrow E$ admits a cross-section if $p^{\prime \prime}: E^{\prime \prime} \rightarrow E$ does so. This proves Theorem 1 .

Now consider the (reduced) Grothendieck group $\widetilde{K}_{R}(B)$ formed from real vector bundles over $B$. If $E$ is a sphere-bundle over $B$ we denote by $[E]$ the class in $\tilde{K}_{R}(B)$ of the associated euclidean bundle. I say that an element $x \in \tilde{K}_{R}(B)$ is of $A$-type if $x=[E]$ where $E$ is a sphere-bundle which admits $A$-structure. By an argument similar to that used to prove Theorem 1 we obtain

THEOREM 2. Let $B$ be a finite-dimensional complex. Let $E$ be an $(n-1)$-sphere bundle over $B$ where $n$ is even and $\operatorname{dim} B \leqq n-4$. Then $E$ admits $A$-structure if $[E]$ is of $A$-type.

From this it is not difficult to show that the elements of $A$-type in $\tilde{K}_{R}(B)$ form a subgroup. Our main result is a determination of this subgroup as follows. Let

$$
T: \quad K_{R}(B) \rightarrow K_{R}(S B)
$$

denote the homomorphism given by taking the tensor product with the class of the canonical line bundle over the real projective line. 
Let $J$ have its standard meaning, as in [1], for example. Then we have

THEOREM 3. An element $x \in \tilde{K}_{R}(B)$ is of $A$-type if and only if $J T(x)=0$.

It is well knownd that the kernel of $T$ coincides with the image of the homomorphism

$$
\tilde{K}_{C}(B) \rightarrow \tilde{K}_{R}(B)
$$

given by taking the underlying real vector bundle. It follows that $T(x)=0$ if and only if $x=[E]$, where $E$ is a sphere-bundle which admits almost-complex structure. It turns out, after a little calculation, that $T$ and $J T$ have the same kernel when $B$ is a sphere or one of the ordinary projective spaces. However, the kernels are different when $B$ is the Cayley projective plane, and so there exists a stable bundle over this 16-dimensional manifold which admits an $A$-structure but not an almost-complex structure.

Outside the stable range there are some fragmentary results. For example it is shown by G. Whitehead [5] that the tangent spherebundle to $S^{n}$ admits an $A$-structure if and only if $n=2$ or 6 . I do not know of any manifold where the tangent bundle admits an $A$-structure but not an almost-complex structure.

An $A$-structure $f$ determined by an almost-complex structure has the properties that (i) $u f=f u$, and (ii) $f^{2}=u$, where $u$ denotes the antipodal map as before. It might be interesting to study conditions for the existence of $A$-structures with one or both of these properties.

The following is an indication of the proof of Theorem 3 . We represent points on the circle $S$ by complex numbers of unit modulus. Let $p: E \rightarrow B$ be as before. Consider the space $\tilde{E}$ formed from $E \times S$ by identifying $(x, z)$ with $(-x,-z)$ for all $x \in E, z \in S$. We fibre $\tilde{E}$ over $B \times S$ with projection $\tilde{p}$ given by $\tilde{p}(x, z)=\left(p x, z^{2}\right)$. The sphere-bundle structure is completed in the obvious way. The vector bundle associated with $\tilde{E}$ is the tensor product of the vector bundle associated with $E$ and the canonical line bundle over $S$.

Let $S_{+}$denote the upper semicircle of $S$. Suppose that $E$ is homotopy-symmetric. Then there exists a map $g: E \times S_{+} \rightarrow E$ such that $p(x, z)=p x$ for all $x \in E, z \in S_{+}$, and such that

$$
g(x, 1)=x=g(-x,-1) .
$$

We extend $g$ to a map $h: E \times S \rightarrow E$ so that $h(x, z)=h(-x,-z)$, for all $x \in E, z \in S$. Then $p k=l \tilde{p}$, where $k: E \rightarrow E$ is induced by $h$ and

- I understand this version of a result of Bott's can be found in D. W. Anderson's unpublished thesis. An independent proof by R. Wood is also unpublished. 
$l: B \times S \rightarrow B$ is the left projection. Since $k$ maps one of the fibres of $\tilde{E}$ homeomorphically onto the corresponding fibre of $E$ it follows that $\tilde{E}$ is fibre-homotopically equivalent to $l^{*}(E)$. In terms of $K$-theory this implies that $T([E])$ lies in the kernel of $J$. When $E$ is a stable bundle the essential steps of this argument are reversible and so, after using Theorem 1, we arrive at Theorem 3 as asserted. Full details are given in [3].

These results can be extended to (right) vector bundles over $F$, where $F$ denotes the field of real numbers, complex numbers or quaternions. Vector spaces over $F$ are endowed with the usual inner product (see $\$ 20$ of [4], for example) so that orthogonality is defined. Let $E$ be a sphere-bundle over $B$ with structural group the orthogonal group in the real case, the unitary group in the complex case, the symplectic group in the quaternionic case. By an $A$-structure on $E$ I mean a fibre-preserving map $f: E \rightarrow E$ such that $f x$ is orthogonal to $x$, in the appropriate sense, for all $x \in E$. Let $D$ denote the unit ball consisting of elements $z \in F$ such that $|z| \leqq 1$, and let $S$ denote the unit sphere, where $|z|=1$. I describe $E$ as homotopy-symmetric if there exists a map $h: E \times D \rightarrow E$ such that

(i) $p h(x, z)=p x \quad(x \in E, z \in D)$,

(ii) $h(x, z)=x \cdot z \quad(x \in E, z \in S)$.

By arguments similar to those used in the real case it can be shown that the existence of an $A$-structure implies homotopy-symmetry, and that the converse holds when $B$ satisfies similar conditions to those in Theorem 1.

The notion of $A$-type is extended to $\tilde{K}_{F}(B)$, in the obvious way. There is no difficulty in generalizing Theorem 2. The generalization of Theorem 3 asserts that the elements of $A$-type coincide with the kernel of $J T$, where

$$
T: \tilde{K}_{F}(B) \rightarrow \tilde{K}_{R}\left(B \wedge P_{F}(1)\right)
$$

is defined by taking the tensor product with the canonical left line bundle ${ }^{5}$ over $P_{F}(1)$, the left projective line. Details are given in [3].

We describe an $A$-structure $f: E \rightarrow E$ as equivariant if

$$
f(x z)=f(x) \cdot z \quad(x \in E, z \in S) .
$$

Consider the case when $B$ is a point-space and $E=S_{n}$, the unit sphere in the $n$-dimensional vector space over $F$. An $A$-structure $f$ on $S_{n}$ determines a nonsingular vector field, by taking the tangent at $x$ to the great circle through $f x$. If $f$ is equivariant we obtain an induced

- As defined in $\$ 4$ of [1]. 
nonsingular vector field on the left $(n-1)$-dimensional projective space $S_{n} / S$. Since complex and quaternionic projective spaces have nonzero Euler number it follows that equivariant $A$-structures cannot exist unless $F$ is real.

\section{REFERENCES}

1. M. F. Atiyah, Thom complexes, Proc. London Math. Soc. (3) 11 (1961), 291-310.

2. W. D. Barcus, Note on cross-sections over CW-complexes, Quart. J. Math. Oxford (2) 5 (1954), 150-160.

3. I. M. James, Bundles with special structure. I, Ann. of Math. (to appear).

4. N. E. Steenrod, The topology of fibre bundles, Princeton Univ. Press, Princeton, N. J., 1951.

5. G. W. Whitehead, Note on cross-sections in Stiefel manifolds, Comment. Math. Helv. 37 (1963), 239-240.

OXFORD UNIVERSITY, OXFORD, ENGLAND 